Sharif University of Technology
Scientia Iranica
SCIENTIA
IRAN ICA

\title{
Impact of natural convection and diffusion on variation of oil composition through a fractured porous medium
}

\author{
A. Papi ${ }^{\mathrm{a}}$, A. Mohebbi ${ }^{\mathrm{a}, *}$, Y. Kazemzadeh ${ }^{\mathrm{b}}$ and S.E. Eshraghi ${ }^{\mathrm{c}, \mathrm{d}}$ \\ a. Department of Chemical Engineering, Faculty of Engineering, Shahid Bahonar University of Kerman, Kerman, Iran. \\ b. Department of Petroleum Engineering, Amirkabir University of Technology, Tehran, Iran. \\ c. Institute of Petroleum Engineering, School of Chemical Engineering, College of Engineering, Tehran University, Tehran, Iran. \\ d. Petro Pajouhesh Ahoura (Knowledge Based Company), Fars Science and Technology Park, Shiraz, Iran.
}

Received 23 December 2015; received in revised form 18 June 2016; accepted 29 August 2016

\section{KEYWORDS \\ Convection; \\ Diffusion; \\ Composition \\ variation; \\ Fractured porous \\ medium; \\ Binary mixture.}

\begin{abstract}
To study an oil reservoir, the first step is to determine its fluid composition in both horizontal and vertical directions. In this research, oil composition variation is investigated as a result of diffusion and natural convection in a fractured porous medium. A single-phase binary mixture and a rectangular two-dimensional fractured model with a known temperature profile are used. Governing equations are solved by MATLAB software, numerically. According to the results, thermal diffusion has the greatest impact on the variation of fluid composition, followed by pressure and molecular diffusions, respectively. Moreover, it is understood that even a small convection would change the fluid composition distribution, considerably. Fluids tend to flow through the fracture due to its high permeability; therefore, a counterclockwise convective cell forms in the porous medium.

(C) 2016 Sharif University of Technology. All rights reserved.
\end{abstract}

\section{Introduction}

Carbonate oil reservoirs are mainly fractured with their specific mechanisms and physical nature. In 1963, Warren and Root brought a new chapter in fractured oil reservoir studies [1], followed by many other research studies; however, several aspects are still open to question. Different mechanisms happen in fractured reservoirs, each of which is vital to have reliable simulation results.

Composition variation is crucial from different points of view, including reservoir simulation, oil in place estimation, and field master development plan. Various trends exist in composition distribution of reservoir fluids. Distribution of fluid composition in

*. Corresponding author. Tel./Fax: +98 3432118298 E-mail addresses: Ali.Papiro@gmail.com (A. Papi); amohebbi@uk.ac.ir (A. Mohebbi);

YusefKazemzade@yahoo.com (Y. Kazemzadeh); eshraghi.ipe@ut.ac.ir (S.E.Eshraghi) some reservoirs may vary in vertical direction $[2,3]$, horizontal direction [4], or it may have uniform distribution [5,6]. Mainly, two major phenomena affect the composition variation in oil reservoirs: 1-natural convection, and 2-diffusion. Natural convection, which results in a circulation of compositions via bulk fluid velocity, is due to temperature and concentration gradient (density gradient). Therefore, natural convection would have a pronounced effect on composition variation. Diffusive flux consists of three diffusions: 1 thermal diffusion, 2 pressure diffusion, and 3 molecular diffusion, which are the results of thermal, pressure, and concentration gradients, respectively. Pressure diffusion or gravitational segregation is compositional segregation due to gravity (components weight). This phenomenon is negligible in the horizontal direction [7].

Few number of studies have considered the effect of convection and diffusion together on composition variation in oil reservoirs. Saidi investigated the molecular diffusion effect on different shapes of fractured reservoirs, including cylindrical, spherical, and 
Cartesian geometries [6]. He analytically solved the dimensionless saturation pressure equation for any of these geometries and sketched the results by graphs. More details about Saidi's work are represented in the validation section. Jacqmin investigated the effect of natural convection, gravitational segregation, and molecular diffusion on the performance of oil reservoirs [8]. He, also, studied different conditions, namely zero-titled or titled reservoirs. However, he did not consider thermal diffusion. In his study, Jacqmin stated that composition variation due to the interaction of diffusion and convection could be significant in both horizontal and vertical directions. Riley and Firoozabadi investigated the effect of natural convection and diffusion (thermal, pressure, and molecular diffusions) on composition variation of a rectangular non-fractured porous medium with prescribed linear temperature field [9]. They showed that a small convection might increase the horizontal composition gradient to its maximum allowable state and, then, decay it with an inverse ratio of permeability $(1 / \mathrm{k})$. Ghorayeb and Firoozabadi studied natural convection and diffusion (thermal, pressure, and molecular diffusions) effects through a rectangular fractured porous medium with prescribed linear temperature field, which was the first comprehensive numerical study of composition variation in a fractured oil reservoir [7]. They showed that composition variation occurred only at fracture apertures that were higher than a certain value. They also demonstrated that the surrounding fractures had the main effect on composition variation, and the fluid flow consisted of a loop within the surrounding fracture.

Afterwards, Ghorayeb and Firoozabadi improved their last study for binary, ternary, and multicomponent mixtures $[10,11]$. They concluded that for high permeability values, convectional mass transfer overrode both thermal and pressure diffusions; whereas, for low permeabilities, a ratio was introduced for which in the values greater than 1 , the composition variation was affected only by thermal diffusion, while in values lower than 1, pressure diffusion was pronounced. In 2000, Ghorayeb and Firoozabadi observed a different behavior of compositions segregation in ternary and multicomponent mixtures in the top and bottom of the porous media. Nasrabadi et al. performed two studies, covering convection and diffusion in porous media $[12,13]$. In their first study, the model of Ghorayeb and Firoozabadi was improved with twophase binary and multicomponent mixtures for both homogeneous and heterogeneous porous media (with and without anisotropy). They also showed that at least two convection cells would flow in the gas cap and the oil column. In the second one, they modeled a packed thermo-gravitational column for binary and ternary mixtures using the same numerical $2 \mathrm{D}$ rectangular model as that in the studies before. Their results showed a good agreement with experimental data. Also, towards steady-state condition, an increase in vertical composition variation was observed while horizontal composition variation decreased to nearly zero.

In this study, the fractured system, used by Ghorayeb and Firoozabadi, with some changes in viscosity values is developed. The equations are rewritten and revised in order to decrease couplings and dependency and to increase the accuracy. These equations are numerically discretized and solved using MATLAB software. The system is investigated from different points of view and interesting results are achieved. A brief summary of comparison between this study and recent ones is represented in Table 1. In this study, finer mesh grids and less coupled equations are hired, which lead to more accurate and faster results. As it can be seen in Table 1, fewer studies are focused on fractured media. The fracture model of Ghorayeb and Firoozabadi investigates the effect of fracture parameters (fracture aperture, fracture intensity, and fracture connectivity) on compositional variation; however, in this study, these parameters are held constant and the effect of each phenomenon on composition variation is investigated instead. In this study, the impact and magnitude of each parameter of natural convection, thermal diffusion, molecular diffusion, and pressure diffusion on composition distribution are investigated and the reason for them is explained in detail, both physically and mathematically, which has been left off before.

\section{Porous medium specifications and modeling methodology}

\subsection{Geometry}

A rectangular two-dimensional fractured porous medium with the width of $a$ and height of $b$ is assumed, as shown in Figure 1. Effect of diffusion and convection on composition distribution of this model, of which the fractures are located at surroundings, is investigated. Fractures and matrix have the same porosities, but different permeability values of $k_{f}$ and $k_{m}$, respectively. The model is saturated with a single-phase

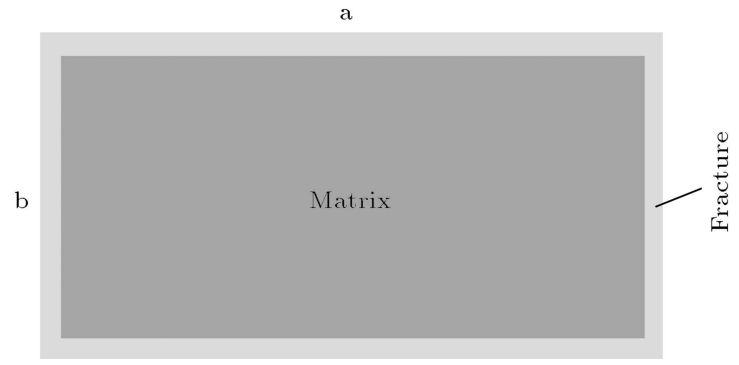

Figure 1. Specifications and geometry of the porous medium (matrix and fracture). 
Table 1. A comparison of different numerical studies on convection and diffusion in different porous media.

\begin{tabular}{|c|c|c|c|c|c|c|c|}
\hline Author(s) & Year & Phase & Mixture & Porous media & Method & $\begin{array}{l}\text { Mesh } \\
\text { grids }\end{array}$ & $\begin{array}{c}\text { Relative } \\
\text { error }\end{array}$ \\
\hline $\begin{array}{l}\text { Ghorayeb and } \\
\text { Firoozabadi [7] }\end{array}$ & 2000 & $\begin{array}{l}\text { Single- } \\
\text { phase }\end{array}$ & Binary & Fractured & $\begin{array}{l}\text { Finite } \\
\text { volume }\end{array}$ & $309 \times 159$ & $\begin{array}{l}10^{-7} \text { units of w } \% \\
\text { (steady state) }\end{array}$ \\
\hline $\begin{array}{l}\text { Ghorayeb and } \\
\text { Firoozabadi [11] }\end{array}$ & 2000 & $\begin{array}{l}\text { Single- } \\
\text { phase }\end{array}$ & $\begin{array}{l}\text { Ternary and } \\
\text { multicomponent }\end{array}$ & Homogeneous & $\begin{array}{l}\text { Volume- } \\
\text { balance }\end{array}$ & $301 \times 41$ & Until steady state \\
\hline $\begin{array}{l}\text { Ghorayeb and } \\
\text { Firoozabadi [10] }\end{array}$ & 2001 & $\begin{array}{l}\text { Single- } \\
\text { phase }\end{array}$ & Binary & Homogeneous & $\begin{array}{l}\text { Finite } \\
\text { volume }\end{array}$ & $301 \times 41$ & $\begin{array}{l}10^{-7} \text { units of w\% } \\
\text { (steady state) }\end{array}$ \\
\hline Nasrabadi et al. [12] & 2006 & $\begin{array}{l}\text { Two- } \\
\text { phase }\end{array}$ & $\begin{array}{l}\text { Binary and } \\
\text { multicomponent }\end{array}$ & $\begin{array}{l}\text { Homogeneous and } \\
\text { heterogeneous } \\
\text { (with and without } \\
\text { anisotropy) }\end{array}$ & $\begin{array}{l}\text { Finite } \\
\text { difference }\end{array}$ & $41 \times 41$ & $\begin{array}{l}10^{-7} \text { units of w } \% \\
\text { (steady state) }\end{array}$ \\
\hline Nasrabadi et. al. [13] & 2006 & $\begin{array}{l}\text { Single- } \\
\text { phase }\end{array}$ & $\begin{array}{l}\text { Binary and } \\
\text { ternary }\end{array}$ & Packed column & $\begin{array}{l}\text { Volume- } \\
\text { balance }\end{array}$ & $21 \times 1001$ & Until steady state \\
\hline This work & 2016 & $\begin{array}{l}\text { Single- } \\
\text { phase }\end{array}$ & Binary & Fractured & $\begin{array}{l}\text { Finite } \\
\text { difference }\end{array}$ & $901 \times 451$ & $\begin{array}{l}10^{-7} \text { units of } \mathrm{w} \% \\
\text { (steady state) }\end{array}$ \\
\hline
\end{tabular}

binary mixture of $C_{1}$ (methane) $/ n C_{4}$ (normal-butane). It is assumed that the model experiences a prescribed thermal gradient via impervious rock boundaries [7].

\subsection{Equations}

The physical model is changed into a mathematical model by writing the governing equations. Governing equations are as follows:

1. Conservation equations of mass and species;

2. Darcy's law;

3. Conservation equation of energy.

Because of the assumed prescribed temperature field, it is not required to solve the last equation. To determine the temperature field, some assumptions are considered, which finally lead us to Eq. (1) as the temperature field of the model. These assumptions include:

1. The conductive flow of heat is much greater than the convective flow;

2. The thermal diffusivity is very large compared with the molecular diffusion coefficient [7].

These assumptions lead us to the equation of $T=$ $T x x+T y y+c$. Considering the temperature of the model's center as $T_{0}$, which is the temperature of sample taken from discovering well, we have:

$$
T=T x(x-a / 2)+T y(y-b / 2)+T_{0} .
$$

Except for the temperature field, another simplifying assumption in this study is Oberbeck-Boussinesq approximation, which is valid in the range of temperature, pressure, and compositions used here [14]. Using this assumption and except for the buoyancy term $(\rho g)$, the density is assumed to be constant and equal to $\rho_{0}$ (center-model density value). The density value for the buoyancy term can be calculated as follows:

$$
\rho(T, w)=\rho_{0}\left[1-\beta_{T}\left(T-T_{0}\right)-\beta_{w}\left(w-w_{0}\right)\right] .
$$

The validity of this equation is demonstrated by Ghorayeb and Firoozabadi (2000) by plotting densities calculated from Peng-Robinson Equation Of State (PREOS) versus temperature and mole fraction in the ranges of temperature, pressure, and compositions used in this study. $\beta_{T}=\left(-1 / \rho_{0}\right)\left(\frac{\partial \rho}{\partial T}\right)$ and $\beta_{w}=$ $\left(-1 / \rho_{0}\right)\left(\frac{\partial \rho}{\partial w}\right)$ are thermal expansion coefficient and compositional expansion coefficient, respectively. Their values are constant and equal to the slope of the abovementioned plots [7]. The index 0 represents the values corresponding to the sampling well.

Conservation equations of mass and species are as follows:

$$
\begin{aligned}
& \nabla . v=0, \\
& \rho_{0} \frac{\partial w}{\partial t}+\rho_{0} v \cdot \nabla w+\nabla . J=0,
\end{aligned}
$$

in which $w, v$, and $J$ are weight fraction of methane, velocity vector, and diffusive mass flux of methane. $J$ is equal to:

$$
J=C^{w} \nabla w+C^{p} \nabla p+C^{T} \nabla T
$$

where $C^{w}, C^{p}$, and $C^{T}$ are molecular, pressure, and thermal diffusion coefficients, respectively. These 
coefficients because of their insignificant changes are assumed to be constant [10]. In binary mixtures, compositions are not independent. That is why it is not necessary to equate the conservation equation for the heavier component, $n$-butane. Diffusive mass flux of $n$-butane equals $-J$ and its weight fraction is equal to $(1-w)$ [7]. Substituting $J$ in Eq. (4) results in the following equation:

$$
\rho_{0} \frac{\partial w}{\partial t}+\rho_{0} v \cdot \nabla w+C^{w} \nabla^{2} w+C^{p} \nabla^{2} p+C^{T} \nabla^{2} T=0 .
$$

The bulk velocity is calculated using Darcy's law:

$$
v=-\frac{k}{\mu \Phi}\left\{\nabla p+\rho_{0}\left[1-\beta_{T}\left(T-T_{0}\right)-\beta_{w}\left(w-w_{0}\right)\right] g j\right\},
$$

where $p, k, \mu, \Phi$, and $j$ are pressure, permeability, fluid viscosity, reservoir porosity, and upward vertical unit vector, respectively. By substituting Darcy's equation in Eq. (3) and assuming that $\frac{k}{\mu \Phi}$ is constant, pressure equation is achieved as:

$$
\nabla^{2} p=\rho_{0} g\left(\beta_{T} \frac{\partial T}{\partial y}+\beta_{w} \frac{\partial w}{\partial y}\right) .
$$

To increase the accuracy of numerical calculations, $\nabla^{2} p$ in Eq. (6) is replaced with its value from Eq. (8). In fact, the coupling of pressure in Eq. (6) is extracted. This equation becomes pressure-independent and is only calculated using velocity and temperature. In other words:

$$
\begin{aligned}
\rho_{0} \frac{\partial w}{\partial t} & +\rho_{0} v \cdot \nabla w+C^{w} \nabla^{2} w \\
& +C^{p} \rho_{0} g\left(\beta_{T} \frac{\partial T}{\partial y}+\beta_{w} \frac{\partial w}{\partial y}\right)+C^{T} \nabla^{2} T=0
\end{aligned}
$$

Generally, there are three types of boundary conditions. In the first type, the dependent variable is specified at the boundary. If we consider $f$ as a function of $x$ and $y$ in a 2D Cartesian problem and $u$ as the dependent variable, we have $u=f(x, y)$ for the $\mathrm{BC}$ of the first kind. In the second type of boundary condition, the normal derivative of dependent variable is specified on the boundary, i.e. $\nabla u . n=f(x, y)$ or $\partial u / \partial n=f(x, y)$, where $n$ is the normal vector. In the third kind, there is a linear combination of the first and second types, i.e. $u+\beta \frac{\partial u}{\partial n}=f(x, y)$, where $\alpha$ and $\beta$ are constants. The first kind of boundary condition is called Dirichlet boundary condition, the second kind is known as Neumann boundary condition, and the third kind is Robins boundary condition. Boundary conditions in this study are of the second kind (Neumann) and the third kind (Robins).

Boundary conditions are given in Figure 2. It is noted that boundaries are bounded and impervious,

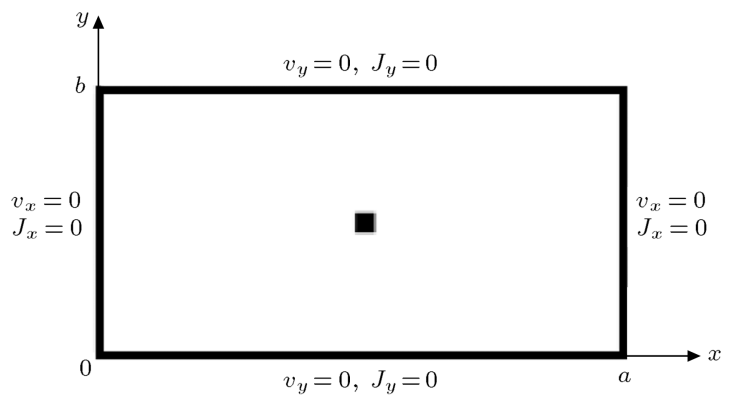

Figure 2. Boundary conditions and location of the discovering well.

and, hence, velocities and diffusion in these points are zero. The above-mentioned sentence can be written mathematically as follows:

$$
\begin{array}{r}
J . n=0, \quad @ x=0, a \text { and } y=0, b, \\
v . n=0 \quad\left(v_{x} \text { and } v_{y}=0\right), \\
\quad @ x=0, a \text { and } y=0, b .
\end{array}
$$

$n$ is unit normal vector. Eq. (10) yields:

$$
\begin{array}{ll}
C^{w} \frac{\partial w}{\partial x}+C^{p} \frac{\partial p}{\partial x}+C^{T} \frac{\partial T}{\partial x}=0, & @ x=0, a, \\
C^{w} \frac{\partial w}{\partial y}+C^{p} \frac{\partial p}{\partial y}+C^{T} \frac{\partial T}{\partial y}=0, & @ y=0, b,
\end{array}
$$

and Eq. (11) yields:

$$
\begin{gathered}
\frac{\partial p}{\partial x}=0, \quad @ x=0, a, \\
\frac{\partial p}{\partial y}=-\rho_{0} g\left[1-\beta_{T}\left(T-T_{0}\right)-\beta_{w}\left(w-w_{0}\right)\right], \\
@ y=0, b .
\end{gathered}
$$

Eqs. (14) and (15) are, respectively substituted in the boundary conditions of Eqs. (12) and (13), to make them pressure-independent. Therefore, we have the following equations:

$$
\begin{aligned}
& C^{w} \frac{\partial w}{\partial x}+C^{T} \frac{\partial T}{\partial x}=0, \quad @ x=0, a, \\
& C^{w} \frac{\partial w}{\partial y}-C^{p} \rho_{0} g\left[1-\beta_{T}\left(T-T_{0}\right)-\beta_{w}\left(w-w_{0}\right)\right] \\
&+C^{T} \frac{\partial T}{\partial y}=0, \quad @ y=0, b .
\end{aligned}
$$

Eqs. (14) and (15) are boundary conditions for Eq. (8), and Eqs. (16) and (17) are boundary conditions for Eq. (9) with initial guesses of $W_{0}$ and $P_{0}$. To solve these equations, it is needed to find the final value of each 
Table 2. Physical properties and dimensions of the model.

\begin{tabular}{clcl}
$a$ & $30 \mathrm{~m}$ & $C^{w}$ & $-1.55 \times 10^{-7} \mathrm{~kg} / \mathrm{m} . \mathrm{s}$ \\
$b$ & $15 \mathrm{~m}$ & $C^{p}$ & $-7.85 \times 10^{-16} \mathrm{~kg} / \mathrm{m} . P a . s$ \\
$X_{0}$ & 0.2 & $C^{T}$ & $1.71 \times 10^{-9} \mathrm{~kg} / \mathrm{m} . \mathrm{s} . \mathrm{K}$ \\
$P_{0}$ & $1.1 \times 10^{7} \mathrm{~Pa}$ & $T_{x}$ & $0.1 \mathrm{~K} / 30 \mathrm{~m}$ \\
$T_{0}$ & $338.5 \mathrm{~K}$ & $T_{y}$ & $-1 \mathrm{~K} / 15 \mathrm{~m}$ \\
$\rho_{0}$ & $495.3 \mathrm{~kg} / \mathrm{m}^{3}$ & $\Phi$ & 0.25 \\
$k_{m}$ & $1 \mathrm{md}$ & $\mu$ & 0.2 c.p. \\
\hline
\end{tabular}

variable at one reservoir point. These values are taken from discovering well samples, namely $T_{0}, P, W_{0}$, and $X_{0}$ (mole fraction); moreover, they, alongside physical properties of the reservoir and model dimensions, are shown in Table 2. In this study, it is assumed that the discovering well is model-center.

Permeability of fractures $\left(k_{f}\right)$ is calculated using the following equation, which makes it related to fracture aperture $\left(f_{a}\right)[7]$ :

$$
k_{f}=10^{5} f_{a}^{2},
$$

where $f_{a}$ is in $\mathrm{mm}$ and $k_{f}$ is in md. Fracture aperture, in this study, is equal to $0.1 \mathrm{~mm}$. Hence, the permeability of fracture is equal to $1000 \mathrm{md}$.

\subsection{Solution procedure}

Darcy's law and Eqs. (8) and (9), along with their boundary conditions, Eqs. (14) to (17), are numerically discretized using finite-difference method and solved using MATLAB. Because the fracture thickness was very less than the reservoir dimension, a non-uniform meshing was used. The shape of mesh grids is shown in Figure 3(a). $901 \times 451$ mesh grids are used for calculations. To investigate effect of the mesh size on numerical results, different simulations with different grid sizes were done. It was found that the applied mesh grid was the optimal grid size both for accuracy and run time. The term "optimal" refers to the highest accuracy and the lowest run time. Error term for weight fraction is $10^{-7}$ units relative error. It is noticeable that indirect solution is time-consuming and inappropriate since there are lots of calculations.

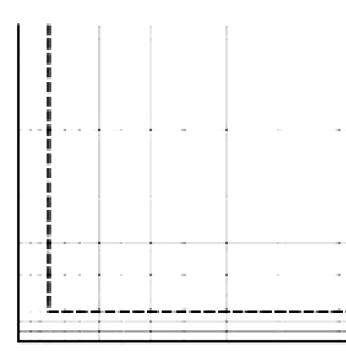

(a)

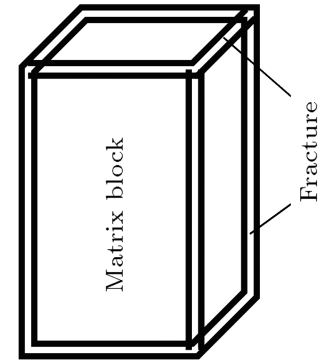

(b)
Figure 3. (a) System mesh grids. (b) Saidi's slab model.
Hence, the direct method of Gaussian elimination is used here $[15,16]$.

\subsection{Validation of the results}

Since the 2D model was insulated from pressure in one dimension $\left(\frac{\partial p}{\partial x}=0\right)$, we used the analogous Saidi's [6] model for slabs, Figure 3(b), to validate our approach. A dimensionless 1D diffusion equation of a slab is as follows:

$$
\frac{\partial^{2} P_{D}}{\partial X^{2}}-\frac{\partial P_{D}}{\partial T_{D}}=0
$$

in which, $P_{D}$ is dimensionless saturation pressure and $X$ and $T_{D}$ are, respectively, dimensionless length and time as follows:

$$
T_{D}=D_{e} \frac{t}{c^{2}}, \quad \text { and } \quad X=\frac{x}{c},
$$

in which, $D_{e}$ is effective diffusion coefficient (diffusion coefficient in porous media) and $c$ is the distance between slab midpoint and boundaries. Boundary conditions are as follows:

$$
\begin{array}{ll}
\frac{\partial P_{D}}{\partial X}=0, & @ X=0, \\
P_{D}=T_{D}, & @ X=1, \\
P_{D}=0, & @ T_{D}=0 .
\end{array}
$$

This dimensionless pressure equation is solved using the method used in this study (Gaussian elimination) at different times and at the slab midpoint $(X=0)$, and a very good match is obtained compared to Saidi's analytical results. Figure 4 demonstrates this claim.

\section{Results and discussion}

Because of the thermal field, pressure gradient, concentration gradient, and density gradient in the reservoir, thermal diffusion, gravitational segregation, molecular

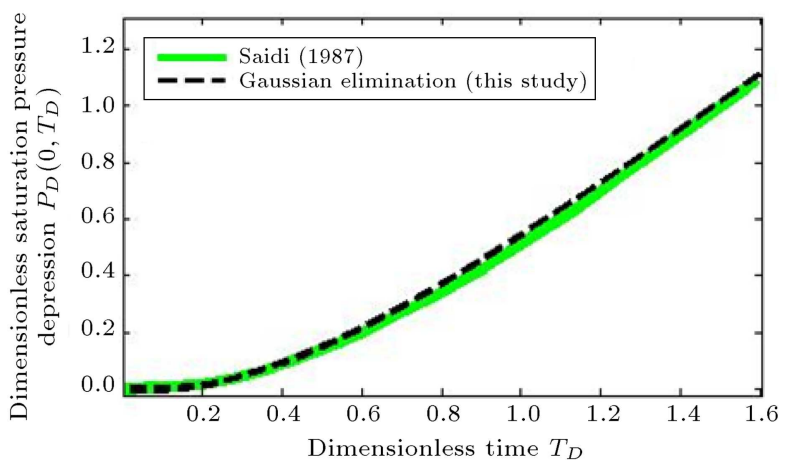

Figure 4. Comparison of Saidi's slab [6] analytical solution and numerical results of the applied method in this study for dimensionless saturation pressure depression at the center of the slab versus dimensionless time. 


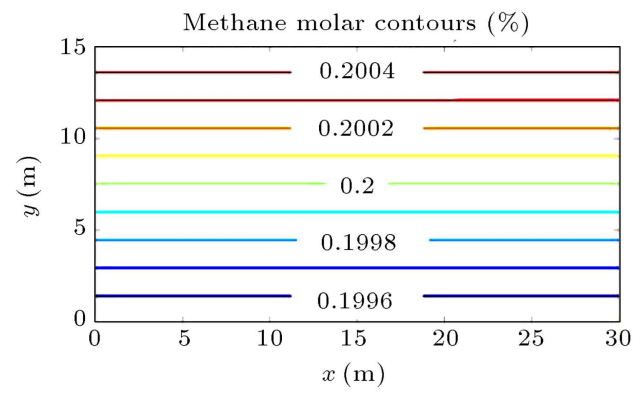

(a)

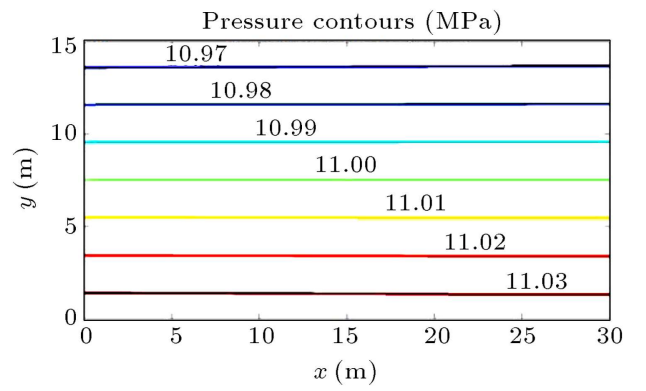

(b)

Figure 5. (a) Methane molar contours for the case $C^{T}=0$ and convection-free system. (b) Methane molar contours for the case $C^{p}=0$ and convection-free system.

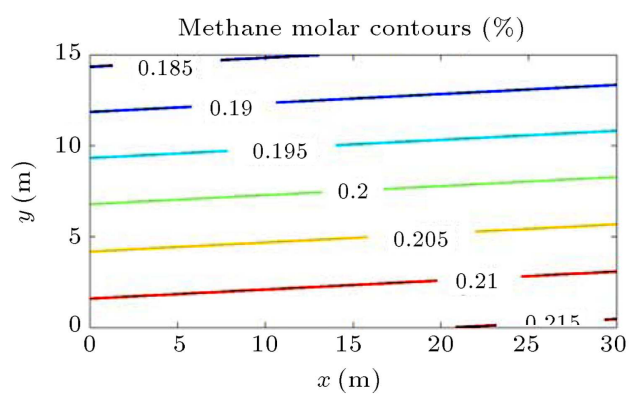

(a)

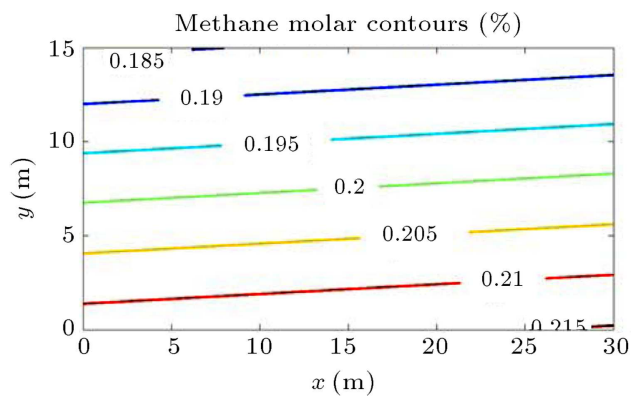

(b)

Figure 6. (a) Methane molar contours for the case $C^{p}=0$ and convection-free system. (b) Methane molar contours (convection-free system $(k=0)$ ).

diffusion, and natural convection are established respectively. These phenomena change the fluid composition. The effect of the mentioned phenomena on the compositional distribution of the reservoir fluid is studied.

To investigate the effect of molecular and pressure diffusions, the values of thermal diffusion coefficient and permeability coefficient are assigned to zero. As shown in Figure 5(a), compositional distribution becomes a zero-inclined straight line. Pressure distribution through the reservoir, also, has such a profile since both boundary conditions of pressure equation in the $x$-direction have a form of $\frac{\partial p}{\partial x}=0$, and the reservoir is pressure insulated in this direction. The pressure profile for this case is shown in Figure 5(b). Hence, it can be found out that pressure diffusion overrides the molecular diffusion. Pressure diffusion would change the composition distribution in the direction of pressure change and yield a zero-inclined distribution. Pressure diffusion or gravitational segregation always tends to settle down the heavier component of the reservoir fluid and keep light component at the top. Thus, it is expected that the reservoir would be richer than light component at the top, and vice versa; or, systematically speaking, reservoir becomes richer than light component from bottom to top. Figure 5(a) bolds the prominence of pressure diffusion.

At this step, to compare the thermal and molecular diffusions, values of pressure diffusion coefficient and permeability coefficient are set to zero. In this case, while pressure BCs still have the same form as before, the weight fraction BCs take the Neumann forms of: $\frac{\partial w}{\partial x}=-\frac{C T}{C w} T x$ and $\frac{\partial w}{\partial y}=-\frac{C^{T}}{C^{w}} T y$, which are multiples of temperature gradients and yield a straight line for composition profile, as shown in Figure 6(a). The temperature profile through the reservoir, also, has the same profile (Eq. (1)). Thus, it could be concluded that the composition of weight fraction takes its effect from temperature profile, meaning that thermal diffusion overrides molecular diffusion and applies changes in fluid composition in the direction of temperature variation to result in an inclined profile. Thermal diffusion always tends to keep the light components at the hotter spots of the reservoir and the heavier components at the colder area. Considering the fact that the reservoir bottom is hotter than the upper zones, it is expected that the lighter components would be more at the bottom and less at the top (vice versa for the heavier components). According to Figure 6(a), it is an implication of thermal diffusion prominence.

In the next case, convection is neglected (by setting $k$ equal to zero) to compare three kinds of diffusion. Again, in this case, pressure BCs have the same form as before but the weight fraction BCs take the Neumann form of:

$$
\frac{\partial w}{\partial x}=-\frac{C T}{C w} T x
$$


in the $x$-direction and the Robins form of:

$$
\begin{gathered}
C^{w} \frac{\partial w}{\partial y}+C^{p} \rho_{0} g \beta_{w} w=-C^{T} T_{y}+C^{p} \rho_{0} g[1 \\
\left.-\beta_{T}\left(T-T_{0}\right)\right]+C^{p} \rho_{0} g \beta_{w} W_{0}
\end{gathered}
$$

in the $y$-direction. Therefore, three terms are added to the weight fraction $\mathrm{BCs}$ in the $y$-direction with respect to the last case. While $C^{P}$ is multiplied in all these three terms, a straight line is still resulted. Regarding Figure 6(b), composition distribution is almost the same as that in Figure 6(a) in which thermal diffusion is the prominent phenomenon. Thus, it could be said that pressure diffusion terms are negligible with respect to thermal diffusion term $\left(C^{T} T_{y}\right)$ and the composition of weight fraction takes its effect from the thermal diffusion, meaning that thermal diffusion overrides molecular and pressure diffusions, no matter the BC is Neumann or Robins. The effect of pressure diffusion and thermal diffusion on composition variation is in contrast, i.e. the pressure diffusion tends to settle down heavier components, while thermal diffusion tends to do the same for lighter components at the bottom (reverse trends for topper zones). Figure 7(a) illustrates the density contours. Despite the presence of gravitational segregation in the reservoir, density at the top is greater than its value at the bottom, which is again an implication of thermal diffusion prominence. Methane is richer at the bottom of the reservoir, and it is said that methane in binary mixtures tends to be segregated into the hotter spots of the reservoir.

To investigate all phenomena, the recent case is repeated by taking the permeability values of fracture and matrix rock into account. A great composition variation is observed and the profile is changed from linear to curved, as presented in Figure 7(b). According to Ghorayeb and Firoozabadi (2000) [7], permeability of fractures that are lower than $0.25 \mathrm{~mm}$ does not greatly change the composition variation. Hence, in our case $(0.1 \mathrm{~mm})$, fractures do not greatly affect the composition. On the other hand, 1 md permeability of matrix rock is relatively low. Thus, one could say

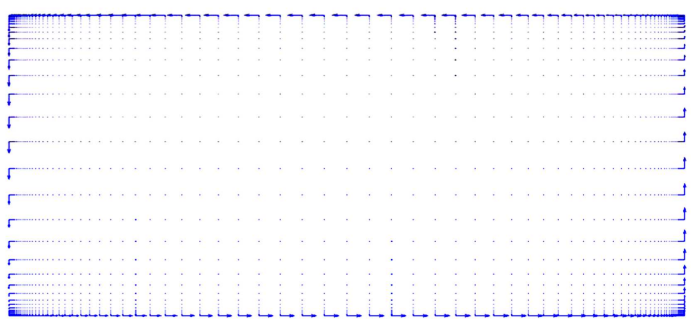

(a)

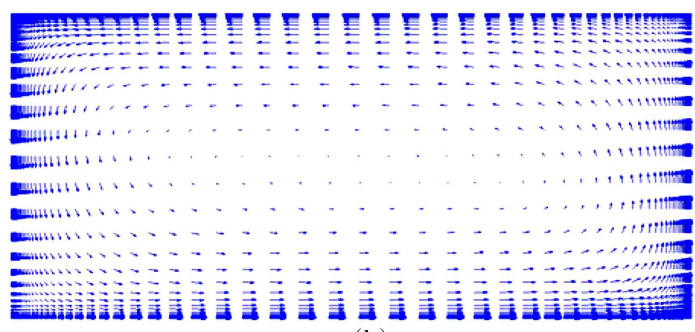

(b)

Figure 8. Velocity vectors of components: (a) When both fracture and homogenous parts play role; and (b) when only velocities of the homogenous part are drawn.

that a small amount of convection has caused this large composition variation.

Figure 8(a) shows velocity vectors in the model. Velocity analysis shows an established counterclockwise convective cell in the model. In the presence of fracture velocities, velocity vectors of the homogenous part will be like a dot. To better demonstrate the trend of velocity contour plots in the homogeneous zone, Figure $8(\mathrm{a})$ is zoomed in such a way that fracture velocities would not be considered. The result is shown in Figure 8(b). As can be seen, the velocity contours take the same counter-clockwise form as that in the fractured zone. The graphs of horizontal velocity of the bottom fracture and the vertical velocity of the left fracture are shown in Figure 9(a) and 9(b), respectively. The existence of high-perm fractures is the reason that the velocities of these parts are much greater than those of the other non-fractured parts, which causes a great portion of the fluid to flow through the fractures. The velocity of fractures increases parabolically up to an extremum in the vicinity of fracture center, and, then, it goes back to zero.

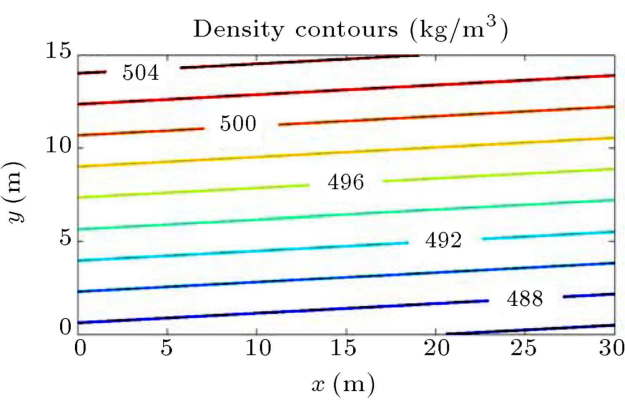

(a)

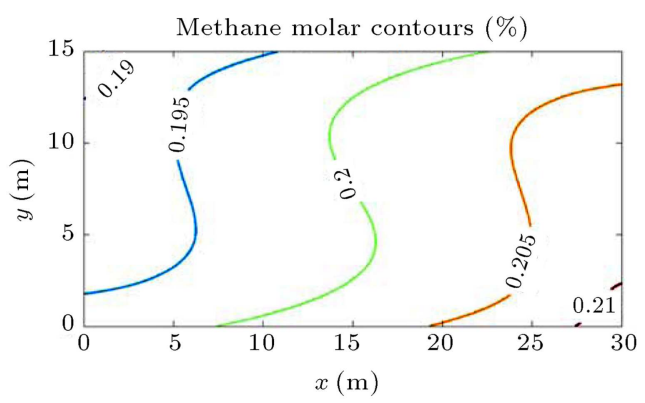

(b)

Figure 7. (a) Density contours (convection-free system $(k=0)$ ). (b) Methane molar contours (convection and diffusion). 


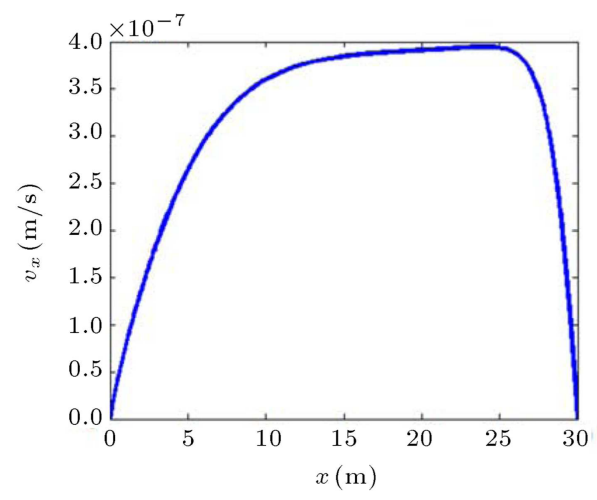

(a)

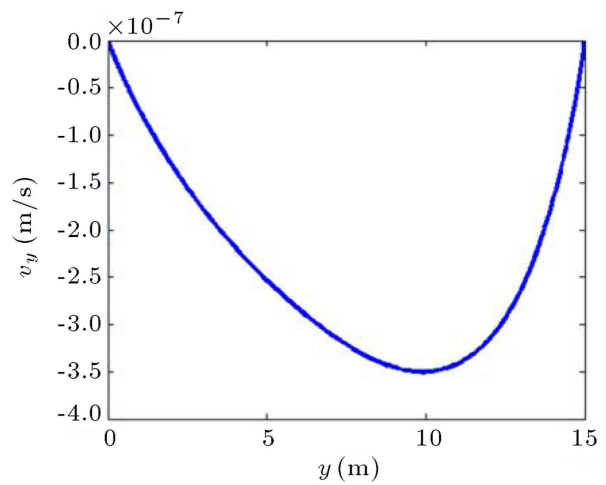

(b)

Figure 9. (a) Horizontal velocity of bottom fracture. (b) Vertical velocity of left fracture.

\section{Conclusions}

In this study, decoupling the pressure from oil conservation equation led us to a faster and more accurate calculation, which resulted in more exact values for the next iterations. According to simulations, the following conclusions could be drawn:

1. Both profile shape and arrangement of the components show that thermal diffusion overrides two other diffusions (i.e., pressure diffusion and molecular diffusion). The profile of oil composition in the convection-free system $(k=0)$ is linear and similar to temperature profile. The behavior of compositions is in such a way that heavier components are segregated at the top and lighter components are segregated at the bottom, which shows that a phenomenon is working against gravitational segregation, i.e. thermal diffusion. The effect of diffusion phenomenon on composition variation is as follows: Thermal diffusion is greater than pressure diffusion and pressure diffusion is greater than molecular diffusion;

2. A small amount of convection causes a large composition variation. For instance, changing the matrix permeability even for $1 \mathrm{md}$ causes a large variation in composition distribution;

3. A counterclockwise convective cell forms in the reservoir, and, in addition, a great portion of the fluid flows through the fractures;

4. In future studies, molecular and pressure diffusions could be neglected because they do not greatly affect composition variation. While, it is not a good idea to neglect thermal diffusion since its effect is significant even in the presence of convection.

\section{Nomenclature}

a Reservoir width, (L), m

$C^{w} \quad$ Coefficient of molecular diffusion in $\mathrm{J}$, $[\mathrm{m} / \mathrm{Lt}], \mathrm{kg} / \mathrm{m} . \mathrm{s}$
Coefficient of pressure diffusion in $\mathrm{J}$, [t], $\mathrm{kg} / \mathrm{m}$.Pa.s

Coefficient of thermal diffusion in $\mathrm{J}$, [m/LTt], kg/m.s.K

$$
\text { Fracture aperture, [L], mm }
$$

Gravity acceleration, $\left[\mathrm{L} / \mathrm{t}^{2}\right], \mathrm{m} / \mathrm{s}^{2}$

Reservoir height, [L], m

$$
\text { Diffusive mass flux, }\left[\mathrm{m} / \mathrm{L}^{2} \mathrm{t}\right], \mathrm{kg} / \mathrm{m}^{2} . \mathrm{s}
$$

$$
\text { Permeability, }\left[\mathrm{L}^{2}\right] \text {, md }
$$

Fracture permeability, $\left[\mathrm{L}^{2}\right]$, md

Matrix permeability, $\left[\mathrm{L}^{2}\right]$, md

\section{Normal vector}

Pressure, $\left[\mathrm{m} / \mathrm{Lt}^{2}\right], \mathrm{Pa}$

Pressure at the reservoir center, $\left[\mathrm{m} / \mathrm{Lt}^{2}\right], \mathrm{Pa}$

Temperature, [T], K

Temperature at the reservoir center, [T], K

$$
\text { Time, [t], s }
$$

Horizontal thermal gradient, [T/L], $\mathrm{K} / \mathrm{m}$

Vertical thermal gradient, $[\mathrm{T} / \mathrm{L}], \mathrm{K} / \mathrm{m}$

$$
\text { Horizontal velocity, }[\mathrm{L} / \mathrm{t}], \mathrm{m} / \mathrm{s}
$$

$$
\text { Vertical velocity, }[\mathrm{L} / \mathrm{t}], \mathrm{m} / \mathrm{s}
$$

Velocity vector, $[\mathrm{L} / \mathrm{t}], \mathrm{m} / \mathrm{s}$

Weight fraction, dimensionless

Weight fraction at the reservoir center, dimensionless

Horizontal axis, [L], m

Vertical axis, [L], m

Upward vertical unit vector

Compositional expansion coefficient, dimensionless Thermal expansion coefficient, $\left[\mathrm{T}^{-1}\right]$, $\mathrm{K}^{-1}$ 
Mole fraction, dimensionless

$\phi \quad$ Porosity, dimensionless

$\mu \quad$ Viscosity, $[\mathrm{m} / \mathrm{Lt}], \mathrm{kg} / \mathrm{m} . \mathrm{s}$

$\rho \quad$ Density, $\left[\mathrm{m} / \mathrm{L}^{3}\right], \mathrm{kg} / \mathrm{m}^{3}$

$\rho_{0}$

Density at $w_{0}$ and $T_{0},\left[\mathrm{~m} / \mathrm{L}^{3}\right], \mathrm{kg} / \mathrm{m}^{3}$

\section{References}

1. Warren, J.E. and Root, P.J. "The behavior of naturally fractured reservoirs", SPEJ, 3(03), pp. 245-255 (1963).

2. Metcalfe, R.S., Vogel, J.L., and Morris, R.W. "Compositional gradients in the Anschutz Ranch east field", SPERE, 3(03), pp. 1-025 (1988).

3. Neveux, A.R., Sakthikumar, S. and Nolray, J.M. "Delineation and evaluation of a north sea reservoir containing near-critical fluids", SPERE, 3(03), pp. 842-848 (1988).

4. Hamoodi, A.N., Abed, A.F. and Firoozabadi, A. "Compositional modeling of two-phase hydrocarbon reservoirs", SPE paper 36244, Presented at Abu Dhabi International Petroleum Exhibition and Conference, Abu Dhabi, UAE (October 13-16, 1996).

5. Lee, S.T. and Chaverra, M. "Modeling and interpretation of condensate banking for the near-critical Cupiagua field" SPE paper 49265, Presented at SPE Annual Technical Conference and Exhibition, New Orleans (September 27-30, 1998).

6. Saidi, A.M., Reservoir Engineering of Fractured Reservoirs (Fundamental and Practical Aspects), Total (1987).

7. Ghorayeb, K. and Firoozabadi, A. "Numerical study of natural convection and diffusion in fractured porous media", SPEJ, 5(01), pp. 12-20 (2000).

8. Jacqmin, D. "The interaction of natural convection and gravity segregation in oil/gas reservoirs", SPE paper 16703, Presented at SPE Annual Technical Conference and Exhibition, Dallas, Texas (September 27-30, 1987).

9. Riley, M.F. and Firoozabadi, A. "Compositional variation in hydrocarbon reservoirs with natural convection and diffusion", AIChE Journal, 44(2), pp. 452-464 (1998).

10. Ghorayeb, K. and Firoozabadi, A. "Features of convection and diffusion in porous media for binary systems", J. Canad. Pet. Tech., 40(02), pp. 21-28 (2001).

11. Ghorayeb, K. and Firoozabadi, A. "Modeling multicomponent diffusion and convection in porous media", SPEJ, 5(2), pp. 158-171 (2000).

12. Nasrabadi, H., Ghorayeb, K. and Firoozabadi, A. "Two-Phase multicomponent diffusion and convection for reservoir initialization", SPEREE, 9(05), pp. 530542 (2006).

13. Nasrabadi, H., Hoteit, H. and Firoozabadi, A. "An analysis of species separation in thermogravitational column filled with porous media", Transport Porous Media, 67(3), pp. 473-486 (2007)

14. Chandrasekhar, S., Hydrodynamic and Hydromagnetic Stability, Courier Corporation (2013).

15. Price, H. and Coats, K. "Direct methods in reservoir simulation", SPEJ, 14(03), pp. 295-308 (1974).

16. Stringer, J.C., Thomas, L.K. and Pierson, R.G. "Efficiency of D4 Gaussian elimination on a vector computer", SPEJ, 25(01), pp. 121-124 (1985).

\section{Biographies}

Ali Papi is an MSc graduate of Chemical Engineering (Reservoir Engineering) from Shahid Bahonar University of Kerman, Kerman, Iran. His interests include numerical simulation and mathematical modelling in chemical and reservoir engineering, fluid mechanics of oil reservoirs, and transport phenomena.

Ali Mohebbi is Professor of Chemical Engineering at Shahid Bahonar University of Kerman, Iran. He received his $\mathrm{PhD}$ from Shiraz University, Shiraz, Iran, in 2002. His research interests include Computational Fluid Dynamics (CFD), porous medium, molecular dynamics simulation, air pollution modeling, and metaheuristics. Besides contributing to a number of books, Dr. Mohebbi has authored and co-authored more than 240 refereed journal and conference publications. He serves on the Editorial Boards of Gas Processing Journal and SOP Transaction on Applied Chemistry. He is a fellow of Iranian Association of Chemical Engineering, Iranian Association of Nanotechnology, Iranian Association of Science and Chemical Technology, and Society of Petroleum Engineering. Dr. Mohebbi has been selected as a Distinguished Researcher at Shahid Bahonar University of Kerman in 2007, 2008, 2009, 2014, and 2015. He has also supervised more than 60 MSc and PhD theses.

Yousef Kazemzadeh is a $\mathrm{PhD}$ candidate at Amirkabir University of Technology, Tehran, Iran. His current interest includes application of nanomaterial in EOR and surface phenomena.

Seyed Ehsan Eshraghi is an MSc graduate from the Institute of Petroleum Engineering of Tehran University, Tehran, Iran. His current interest includes reservoir modeling using fast simulation techniques, fracture analysis, and investigation of the (heavy) oil recovery and asphaltene concerns, particularly in the presence of nanoparticles, which have been demonstrated by several publications. Mr. Eshraghi is also a member of SPE and EAGE. 